OPEN ACCESS

Edited by:

Steven Thomas Leach,

University of New South

Wales, Australia

Reviewed by:

Juan Jovel,

University of Alberta, Canada Renata D'Incà,

University Hospital of Padua, Italy

*Correspondence:

Gary P. Moran

gpmoran@dental.tcd.ie

Specialty section:

This article was submitted to Pediatric Gastroenterology,

Hepatology and Nutrition

a section of the journal

Frontiers in Pediatrics

Received: 22 October 2020 Accepted: 14 December 2020

Published: 21 January 2021

Citation:

Elmaghrawy K, Hussey S and Moran GP (2021) The Oral

Microbiome in Pediatric IBD: A Source

of Pathobionts or Biomarkers?

Front. Pediatr. 8:620254.

doi: 10.3389/fped.2020.620254

\section{The Oral Microbiome in Pediatric IBD: A Source of Pathobionts or Biomarkers?}

\author{
Khalid Elmaghrawy ${ }^{1}$, Séamus Hussey ${ }^{2,3}$ and Gary P. Moran ${ }^{1 *}$ \\ ${ }^{1}$ School of Dental Science, Trinity College Dublin and Dublin Dental University Hospital, Dublin, Ireland, ${ }^{2}$ Department of \\ Paediatrics, University of Medicine and Health Sciences, Royal College of Surgeons in Ireland, Dublin, Ireland, ${ }^{3}$ National \\ Children's Research Centre, Dublin, Ireland
}

The oral cavity is continuous with the gastrointestinal tract and in children, oral health may be closely linked with the overall health of the Gl tract. In the case of pediatric Crohn's disease (CD), oral manifestations are an important clinical indicator of intestinal disease. Recent studies of the microbiome in IBD suggest that translocation of oral microbes to the gut may be a common feature of the microbial dysbiosis which is a signature of both $\mathrm{CD}$ and ulcerative colitis (UC). Murine studies suggest that translocation of oral bacteria and yeasts to the lower Gl tract may trigger inflammation in susceptible hosts, providing a mechanistic link to the development of IBD. Conversely, some studies have shown that dysbiosis of the oral microbiome may occur, possibly as a result of inflammatory responses and could represent a useful source of biomarkers of Gl health. This review summarizes our current knowledge of the oral microbiome in IBD and presents current hypotheses on the potential role of this community in the pathogenesis of these diseases.

Keywords: inflammatory bowel disease, Crohn's disease, ulcerative colitis, microbiome, dysbiosis, inflammation, oral cavity

\section{INTRODUCTION}

In the human body, microbial cells are thought to be at least as numerous as host cells (1-4). The microbiome of the human digestive tract is made up of hundreds of bacterial and fungal species and these microbes harbor 150 times more genes compared to the human genome $(5,6)$. Microbial population densities in this complex consortium reach their maximum values in the colon, with $10^{11}$ bacteria per gram of content (4). The gut microbiota play a critical role in human health and have been implicated in nutrient absorption, mucosal barrier fortification, xenobiotic metabolism, angiogenesis and postnatal intestinal maturation $(7,8)$. Furthermore, the human microbiota stimulates immunity (innate and adapative) and plays an important role in maturation of the immune system (9). Additionally, studies in germ-free mice show that gut microbiota influence body fat deposition, metabolism, and immune function $(10,11)$. Recent studies suggest that IBD is the result of an altered immune response to the gut microbiota (12). Perturbations in the microbiome may lead to dysbiosis which can be defined as "changes to the structure of a microbial community that are detrimental to its host" (13). This may impair important functions of the microbiome, including its ability to resist pathogenic microorganisms. In the oral cavity, microbiome perturbations caused by increased sugar intake or poor oral hygiene may lead to dental caries and inflammatory gum diseases, respectively $(14,15)$. Changes to the human gut microbiome have been linked with the prevalence and severity of several diseases, including cancers, IBD 
and atopic disease $(5,7)$. The role of the microbiome in the pathogenesis of IBD is still hotly debated, but the disease involves a strong inflammatory response that may be triggered by acquired infection or alternatively by dysbiotic changes to the hosts' own microbiome. In the case of $\mathrm{CD}$, the trigger for this breakdown in homeostasis is unknown, but recent evidence suggests that environmental factors, host genetics and possibly oral-gut transit of microorganisms could all play a role in perturbation of the relationsip between the mucosal immune system and the gut microbiota $(16,17)$.

\section{DEVELOPMENT OF THE ORAL AND GUT MICROBIOMES IN CHILDREN}

Most of the microbial biodiversity in the human microbiome can be found in the GI tract, and in particular the oral and gut microbiomes (1). However, in terms of composition, these two communities are very distinct. The oral cavity is dominated by facultative, sugar fermenting organisms (e.g., Streptococcus and Actinomyces spp.) whereas the gut is dominated by a metabolically diverse community of anaerobic bacteria (e.g., Clostridium and Bacteroides spp.) (1, 18). Although these communities have distinct compositions, the level of species richness is similar in both environments and a single individual may harbor over 100 distinct species at each site (1). Interestingly, the oral microbiome exhibits less interindividual variation compared to the gut microbiome (1). The greater interindividual variation observed in gut microbiomes appears to be related to the greater impact of factors such as diet and antibiotic usage on these communities, whereas as the oral microbiome appears to be more resilient to these challenges $(11,19-21)$. Both communities are relatively stable over time in healthy individuals (1).

The development and maturation of the gut microbiome in infancy is influenced by several factors, including mode of delivery, type of feeding and antibiotic usage (22) (Figure 1). Disturbances to this development have been linked to obesity and atopic disease, suggesting that development of this microbiome has a strong impact on immune maturation and metabolism $(23,24)$. Some of this regulatory activity has been related to bacterial metabolites including short chain fatty acids (SCFAs) such as acetate, propionate and butyrate. SCFAs are not only an important source of nutrients for enterocytes but also induce differentiation of regulatory $\mathrm{T}$ cells (Tregs) which have a central role in the suppression of inflammatory and allergic responses (9, $25,26)$. Nutrition also plays an important role in gut microbiome development. In early life, infant breast feeding is associated with reduced biodiversity and high levels of Bifidobacterium species capable of metabolizing human milk oligosaccharides (HMOs) (27). The introduction of solid food later results in an increase in biodiversity and the capacity to metabolize more complex carbohydrates. Children exposed to antibiotics display delayed gut microbiome maturation, especially if administered in the first 12 months of life and this can lead to increased levels of Enterobacteriaceae and Bacteroidetes $(27,28)$. The gut microbiome stabilizes at approximately 3 years of age, but diet continues to have a major influence on composition in childhood and later life. Children consuming western diets rich in fats and low in fiber exhibit increased levels of Bacteroides species, whereas plant and fiber rich diets are associated with Prevotella and Succinivibrio (29-31). In general, fat-rich western diets may lead to reduced biodiversity and lower production of SCFAs, which may impact on the development of inflammatory bowel disease $(32,33)$.

The oral microbiome also develops and matures throughout childhood. The mature oral microbiome is a highly diverse community of up to 700 bacterial species, in addition to the oral fungal mycobiome which is dominated by the yeasts Candida, Malassezia and Saccharomyces spp. (18, 34-36). The development of this oral microbiome begins shortly after birth and recent data shows that this is also influenced by delivery method and infant feeding (37). Diversity increases with tooth eruption and continues to evolve as children move from decidious to permanent dentition $(37,38)$. Sugar intake and

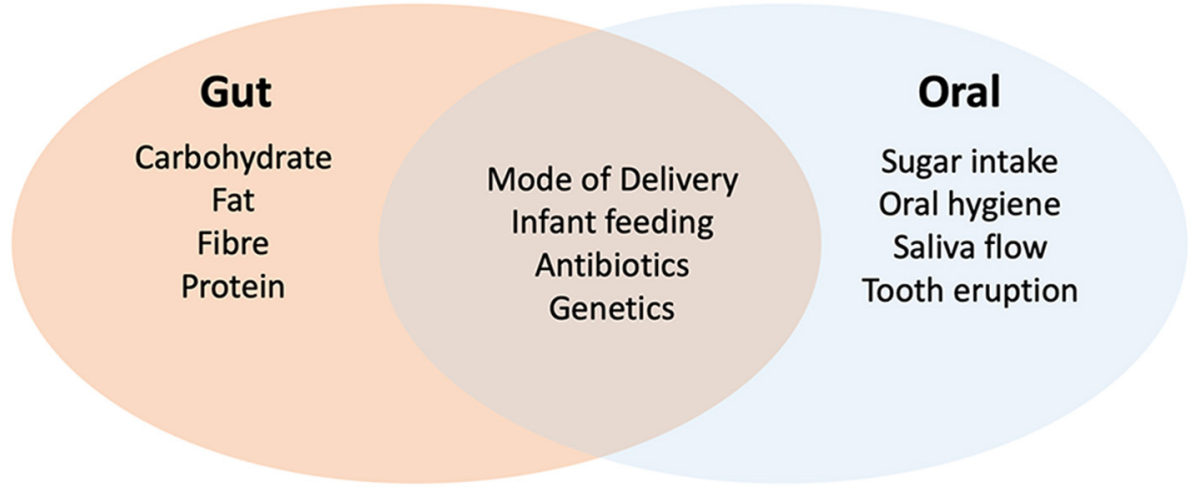

FIGURE 1 | Factors affecting the development of the oral and gut microbiomes in childhood. Evidence shows that both oral and gut microbiomes are influenced by delivery (natural or cesarean), mode of feeding (breast or formula milk) and antibiotics. Genetics may also play a role in colonization. Development of the gut microbiome is heavily influenced by diet whereas sugar intake (especially frequent consumption) has a greater impact on the oral microbiome. In the oral environment, tooth eruption and the level of plaque development (impacted by hygiene and saliva flow) have a major impact on diversity. 
poor oral hygiene have a major impact on the oral microbiome in children $(19,39)$. Sugar intake in particular is linked to increased levels of acid tolerant species such as S. mutans and Lactobacillus spp. $(19,40)$, the former being directly linked with the pathogeneisis of dental caries. Other dietary factors such as vegetables and refined grain servings have more moderate effects $(37,41)$. Antibiotic usage in childhood has been shown to affect the oral microbiome, however studies in adults sugest that the oral microbiome recovers more rapidly from antibiotic exposure compared to gut communities $(20,37)$.

\section{ORAL AND GUT MANIFESTATIONS OF IBD}

Crohn's disease (CD) and Ulcerative colitis (UC) manifest in different ways. CD is typically characterized by granulomatous inflammation that can affect any part of the gastrointestinal tract (GIT) and involve all mucosal layers, while the inflammation in UC is limited to the colon and only affects the mucosa and superficial submucosa (42-44). The variability in location and the transmural nature of CD leads to a wide variety of presentations and in severe cases, patients may present with systemic symptoms, including fever, anorexia, and weight loss $(45,46)$. Inflammation in CD most frequently affects the distal ileum and colon but may occur in any part of the gastrointestinal tract including the mouth (47). The term oral Crohn's disease (OCD) is used to describe patients with intestinal CD who exhibit involvement of the oral cavity with a wide variety of disease-specific oral lesions (48). Many oral lesions have been described in CD patients, including swelling of the lips, buccal mucosal swelling or "cobble-stoning," mucogingivitis, deep linear ulceration, particularly along the buccal gutters, and mucosal tags. Submental lymphadenopathy, perioral erythema with scaling, recurrent buccal abscesses, and angular cheilitis are often observed in patients with CD $(43,48)$. The oral manifestations of IBD are diverse and based on their relationship with $\mathrm{CD}$ activity can be classified as specific (e.g., mucosal swelling) and non-specific oral lesions (e.g., angular cheilitis) (49). Similar oral manifestations have been recorded in different patient cohorts throughout the world (47-52). Although patients with $\mathrm{CD}$ can exhibit oral manifestations, pediatric $\mathrm{CD}$ has no specific clinical manifestations. Some studies have suggested that oral manifestations are a good cutaneous marker of IBD (50) and are useful diagnostic markers (53).

Orofacial granulomatosis (OFG) is a rare chronic disease that can present with symptoms similar to oral Crohn's disease, including lip swelling and oral inflammation (54). Despite the clinical similarity, OFG patients rarely present with clinical signs of intestinal CD and it is not known if the etiology of OFG is related to that of CD (54). OFG patients have a high incidence of atopy and some studies have suggested the involvement of IgE expressing B-cells in the development of the disease, suggesting that this may involve a hypersensitivity reaction $(55,56)$.

\section{THE GUT MICROBIOME IN IBD}

Significant alterations to the gut and oral microbiomes (discussed below) have been identified in patients with IBD, including both CD and UC patients (12, 17, 51, 57-62). Microbial dysbiosis in the gut of IBD patients has been associated with the generation of a chronic inflammatory response which may be exacerbated by a decrease in community members known to produce immunomudulating SCFAs and increased levels of the Enterobacteriaceae $(57,63,64)$. Within the last decade, metagenomic profiling microbiome studies have provided detailed information on this dysbiosis and unexpectedly have identified increased abundance of common oral taxa in the gut microbiomes of patients with IBD (e.g., Veillonella, Haemophilus, Eikenella spp.) whereas many bacteria with important roles in the generation of SCFAs were reduced $(59,62,65)$. An increased $\mathrm{H}_{2} \mathrm{~S}$ producers in IBD patients was also noted by Mottawea et al. (2016), which was linked to a reduced capacity of mitochondria from IBD patients to detoxify $\mathrm{H}_{2} \mathrm{~S}$, suggesting an additional mechanism for microbiome induced inflammation (60). Studies have shown that this dysbiosis is dynamic and is greatest in patients with severe disease and those with a history of antibiotic usage (59). Medications such as infliximab can also influence the degree of dysbiosis (66-68). The degree of dysbiosis has also been linked to host genetics, with NOD2 risk alleles being shown to be associated with the degree of dysbiosis and the levels of Enterobacteriaceae in IBD patients $(69,70)$.

Changes in the fungal mycobiome have also been reported in IBD patients $(71,72)$. Fungi are often overlooked because of their low abundance in the lower GIT of humans, however, fungi account for approximately $13 \%$ of the gut microbial volume (6, 73). Several investigators have shown an increase in the levels of fungi belonging to the Basidiomycota in IBD patients, including the yeasts of the genus Malassezia (74-76). Limon et al. (2019) found that the presence of the Malassezia spp. in CD showed strong linkage to an allele of the CARD9 receptor (CARD9 ${ }^{S 12 N}$ ) associated with CD (76). They went on to demonstrate that $M$. restrica could induce colitis in susceptible mice, providing the first evidence that a member of the normal mycobiome may play a role in the pathogenesis of $\mathrm{CD}$ in at least a subset of patients (76).

In summary, the gut microbiome in pediatric IBD is now well-characterized. Several longitudinal, large cohort studies involving treatment naïve patients have clearly shown that changes to the gut microbiome are intrinsically linked to IBD and are responsive to therapy $(59,62,65)$. This dysbiosis appears to be associated with reduced biodiversity and reduced levels of normal gut microbes with increases in the levels of Enterobacteriaceae and bacterial and yeast species normally associated with the oral cavity (e.g., Veillonella, Haemophilus, Eikenella and Malassezia spp.).

\section{CHANGES IN THE ORAL MICROBIOTA IN PATIENTS WITH CROHN'S DISEASE}

A general feature of the studies described above is the identification of high levels of oral taxa in the gut of IBD patients. The oral microbiome is well-characterized in terms of its role in oral diseases (caries, periodontitis), however its members have also been implicated as contribitory factors in several non-oral diseases such as colorectal cancer, diabettes 
mellitus, cardiovascular disease, bacteremia and preterm birth (77). Relatively few studies have directly investigated the impact of IBD on the oral microbiome in pediatric patients. The first analysis of the oral microbiome in pediatric IBD was carried out using DNA microarrays (51). In this study oral swab samples from the tongue and buccal mucosa of pediatric CD patients on active treatments were examined. Although the species-resolution of DNA microarray analysis is weak compared to more recent sequence based approaches, it was found that the overall microbiome biodiversity of $\mathrm{CD}$ patients was significantly reduced compared to healthy children. The tongue samples from pediatric CD patients showed reduced levels of two phyla (Fusobacteria and Firmicutes) and additionally, $\mathrm{CD}$ patients with oral manifestations were also shown to have a higher level of anti-Saccharomyces cerevisiae antibody (ASCA) (51).

More recently, 16S sequence based approaches have been used to examine the salivary microbiome in IBD. Said et al. (2014) analyzed the salivary microbiome of adult IBD patients $(n=35)$ including both CD and UC patients (61). Significant shifts in the oral microbiome composition of adult CD patients were observed, with higher levels of Prevotella and Veillonella spp. and reduced Streptococcus and Haemophilus spp. (61). Furthermore, a study by Xun et al. (2018) also examined the salivary microbiome in adult IBD patients $(\mathrm{UC}=57$ and $\mathrm{CD}=$ 13) (78). This analysis revealed increased levels of Streptococcus spp. and Enterobacteriaceae in UC patients, increased abundance of Veillonella spp. in CD patients, accompanied by depletion of Prevotella, Neisseria and Haemophilus spp. (78). Zhang et al. (2020) compared the salivary microbiome of CD patients $(n=$ $29)$ with patients in remission $(n=31)$ and showed that active CD was associated with depletion of Neisseria, Haemophilus, Fusobacterium and Porphyromonas spp. compared to those patients in remission (79). Taken together, these sequence based studies show that oral dysbiosis occurs in IBD, although the exact nature of the dysbiosis characterized differs between studies. This may be due to the relatively small patient cohorts examined and the use of diverse anti-inflammatory medications in each group. In addition, in the context of pediatric IBD, none have exclusively focused on children and do not include longitudinal elements to determine if treatment is associated with reversal of dysbiosis. Despite these limitations, most studies concur that the genera Prevotella, Haemophilus and Veillonella are somehow affected in the oral cavity in IBD, which interestingly matches the genera shown to be enriched in the gut microbiota in $\mathrm{CD}$ patients (59). The possible link between changes in the oral microbiome and gut microbiota will be discussed further below.

The relationship between OFG oral CD is also under intense investigation and studies of the oral microbiome may indicate if these diseases have similar etiologies. Recently Goel et al. (2019) examined the salivary microbiome in 261 subjects comparing the oral microbiome of OFG patients and $\mathrm{CD}$ patients (including those with and without OFG) (80). A specific sequence type (oligotype) of $S$. salivarius was found to exhibit increased abundance in individuals with CD or OFG compared to controls and may have promise as an early biomarker of CD (80).

\section{ORAL-GUT MICROBIAL TRANSIT IN IBD?}

Recent studies of the gut microbiota in pediatric IBD patients have shown an increase in $H$. parinfluenza, Veillonellaceae, Neisseriaceae, and Fusobacteriaceae in CD patients (59) and H. parainfluenza and Veillonella spp. in UC patients (62). The increased abundance of these taxa in the gut environment may be the result of oral-gut transit. Despite the barrier of the acidic $\mathrm{pH}$ of the stomach, oral-gut transit of microorganisms is probably commonplace, as studies of probiotic bacteria show that microrganisms can transit from the oral cavity to the gut and remain viable (81). Auchtung et al. (82) also recently showed that yeast in the gut were acquired from the oral cavity and their levels in the gut were associated with dietary consumption (Saccharomyces) or poor oral hygiene (Candida).

Transit of these microorganisms from the oral cavity may disrupt host-microbiome homeostasis in the gut by displacing commensal SCFA producers. Additionally, some studies indicate that these oral taxa may be directly immunogenic. Previous studies have suggested that two of these bacterial taxa, H. parinfluenza and Veillonella spp. induce dendritic cell maturation, with the mature dendritic cells producing cytokines which may drive inflammation (83). Rothia mucilaginosa, a species associated with ulceration by Gevers et al. (59) can produce toxic levels of acetaldehyde in the presence of ethanol, which is a by-product of Enterobacterial fermentations (84). Rengarajan et al. (85) also showed significant antibody responses in the colon of IBD patients to bacteria normally found in the oral cavity including Gemella, Peptostreptococcus, and Streptococcus species.

Experimental evidence for the impact of oral gut transit in IBD patients was reported by Atashari et al. (86), who showed that inoculation of the oral microbiome from IBD children to C57BL/6 (B6) germ-free mice resulted in the accumulation of inflammatory IFN-g+ CD4+ TH1 cells. The gut microbiome of these mice was enriched in Fusobacterium, Veillonella and Klebsiella spp. Subsequent experiments showed that a Klebsiella pneumonia strain (Kp-2H7) recovered from the human salivary microbiota was the primary driver of the inflammatory $\mathrm{TH} 1$ cell responses, with marked accumulation of TH1 cells in the murine colon. These data clearly show that the oral cavity may be a reservoir for pathobionts, and that trasit from the oral cavity to the gut may play a role in inducing inflammation in IBD patients (86).

\section{CONCLUSIONS AND FUTURE DIRECTIONS}

The understandable focus of IBD research to date on the intestinal microbiota has left much to be elucidated regarding the oral microbiome in patients with IBD. Transit of oral microorganisms likely happens in all children, but whether a genetic susceptibility to colonization with pathobionts exists in children with IBD, or whether such taxa are acquired secondarily is unkonwn. It is our hypothesis that oral-gut 


\section{Oral Microbiome:}

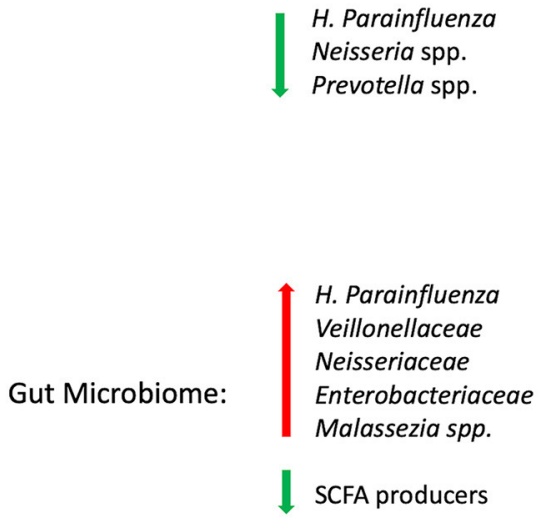

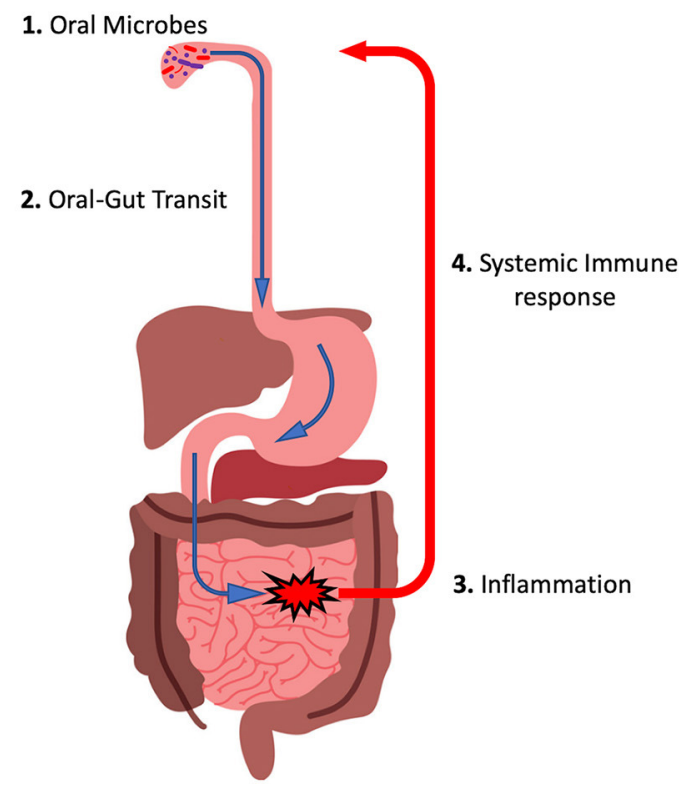

FIGURE 2 | Cartoon diagram summarizing the oral-gut axis hypothesis in pediatric IBD. (1) Microbes in the oral cavity transit through the GI tract (2). In susceptible individuals this may induce inflammatory reactions (3) and displace health promoting SCFA producers. (4) Systemic immune responses to these invading microbes may induce inflammatory reactions that could be involved in generating oral manifestations and altering the abundance of these taxa in the oral microbiome. Red arrows indicate taxa with increased abundance in IBD and green arrows those with reduced abundance. See text for details.

transit of Veillonella, Klebsiella or Malassezia spp. could trigger pathobiont-specific systemic responses that could induce inflammatory responses in the oral cavity resulting in oral manifestations of IBD (Figure 2). These systemic reactions may also account for the altered abundance of these species in the oral cavities of IBD patients. The specific nature of this response may make the oral cavity a useful source of biomarkers to diagnose and monitor treatment outcomes in IBD patients. Much of the existing data on the oral microbiome in IBD has come from patients already on various treatments. The effects of chronic treatments or periods of increased disease activity on oral microbial profiles have not yet been studied in sufficient detail. The "holy grail" of biomarker development based on oral sampling remains some time away, and yet would provide a more convenient, accessible and acceptable source for

\section{REFERENCES}

1. Human Microbiome Consortium, Huttenhower C, Gevers D, Knight R, Abubucker S, Badger JH, et al. Structure, function and diversity of the healthy human microbiome. Nature. (2014) 486:207. doi: 10.1038/nature11234

2. Gevers D, Knight R, Petrosino JF, Huang K, McGuire AL, Birren BW, et al. The Human microbiome project: a community resource for the healthy human microbiome. PLoS Biol. (2014) 10:e1001377. doi: 10.1371/journal.pbio.1001377

3. Dave M, Higgins PD, Middha S, Rioux KP. The human gut microbiome: current knowledge, challenges, and future directions. Transl Res J Lab Clin Med. (2012) 160:246-57. doi: 10.1016/j.trsl.2012.05.003 patients and clinicians than current blood or stool specimenbased assays.

\section{AUTHOR CONTRIBUTIONS}

$\mathrm{KE}, \mathrm{GPM}$, and SH all contributed to the literature survey, writing, and editing of the final review.

\section{FUNDING}

KE was supported by a scholarship from the Libyan Ministry of Education and Research. GPM was supported by the Irish Health Research Board (Grant No. ILP-POR-2019-030). SH was supported by the Irish National Children's Research Centre (Grant Code C/18/2). 
8. Hooper LV, Wong MH, Thelin A, Hansson L, Falk PG, Gordon JI. Molecular analysis of commensal host-microbial relationships in the intestine. Science. (2001) 291:881-4. doi: 10.1126/science.291.5505.881

9. Arpaia N, Campbell C, Fan X, Dikiy S, Veeken J van der, deRoos P, et al. Metabolites produced by commensal bacteria promote peripheral regulatory T-cell generation. Nature. (2013) 504:451-5. doi: 10.1038/nature12726

10. Bäckhed F, Manchester JK, Semenkovich CF, Gordon JI. Mechanisms underlying the resistance to diet-induced obesity in germ-free mice. Proc National Acad Sci. (2007) 104:979-84. doi: 10.1073/pnas.0605374104

11. David LA, Maurice CF, Carmody RN, Gootenberg DB, Button JE, Wolfe BE, et al. Diet rapidly and reproducibly alters the human gut microbiome. Nature. (2014) 505:559-63. doi: 10.1038/nature12820

12. López RL, Burgos MJG, Gálvez A, Pulido RP. The human gastrointestinal tract and oral microbiota in inflammatory bowel disease: a state of the science review. APMIS. (2016) 125:3-10. doi: 10.1111/apm.12609

13. Miles J, Holt JF, Handelsman J. Allies and adversaries: roles of the microbiome in infectious disease. Microbe Mag. (2015) 10:370-4. doi: $10.1128 /$ microbe. 10.370 .1

14. Belda-Ferre P, Alcaraz LD, Cabrera-Rubio R uacute l, Romero H eacute ctor, $\mathrm{n}$ Soro AS oacute, Pignatelli M, et al. The oral metagenome in health and disease. ISME J. (2011) 6:46. 56. doi: 10.1038/ismej.2011.85

15. Teles R, Teles F, Frias-Lopez J, Paster B, Haffajee A. Lessons learned and unlearned in periodontal microbiology. Periodontol 2000. (2013) 62:95-162. doi: $10.1111 /$ prd.12010

16. McGovern DPB, Gardet A, Törkvist L, Goyette P, Essers J, Taylor KD, et al. Genome-wide association identifies multiple ulcerative colitis susceptibility loci. Nat Genet. (2010) 42:332-7. doi: 10.1038/ng.549

17. Lloyd-Price J, Arze C, Ananthakrishnan AN, Schirmer M, Avila-Pacheco J, Poon TW, et al. Multi-omics of the gut microbial ecosystem in inflammatory bowel diseases. Nature. (2019) 569:655-62. doi: 10.1038/s41586-019-1237-9

18. Dewhirst FE, Chen T, Izard J, Paster BJ, Tanner ACR, Yu W-H, et al. The human oral microbiome. J Bacteriol. (2010) 192:5002-17. doi: 10.1128/JB.00542-10

19. Rosier BT, Marsh PD, Mira A. Resilience of the oral microbiota in health: mechanisms that prevent dysbiosis. J Dent Res. (2017) 97:371-80. doi: $10.1177 / 0022034517742139$

20. Zaura E, Brandt BW, Mattos MJT de, Buijs MJ, Caspers MPM, Rashid M$\mathrm{U}$, et al. Same exposure but two radically different responses to antibiotics: resilience of the salivary microbiome versus long-term microbial shifts in feces. mBio. (2015) 6:e01693-15. doi: 10.1128/mBio.01693-15

21. Zaura E, Nicu EA, Krom BP, Keijser BJF. Acquiring and maintaining a normal oral microbiome: current perspective. Front Cell Infect Microbiol. (2014) 4:85. doi: $10.3389 /$ fcimb.2014.00085

22. Bokulich NA, Chung J, Battaglia T, Henderson N, Jay M, Li H, et al. Antibiotics, birth mode, and diet shape microbiome maturation during early life. Sci Transl Med. (2016) 8:343ra82. doi: 10.1126/scitranslmed.aad7121

23. Dogra S, Sakwinska O, Soh S-E, Ngom-Bru C, Brück WM, Berger B, et al. Rate of establishing the gut microbiota in infancy has consequences for future health. Gut Microbes. (2015) 6:321-5. doi: 10.1080/19490976.2015.1078051

24. Korpela K, Vos W de. Antibiotic use in childhood alters the gut microbiota and predisposes to overweight. Microb Cell. (2016) 3:296-8. doi: 10.15698/mic2016.07.514

25. Inan MS, Rasoulpour RJ, Yin L, Hubbard AK, Rosenberg DW, Giardina C. The luminal short-chain fatty acid butyrate modulates NF- $\kappa$ B activity in a human colonic epithelial cell line. Gastroenterology. (2000) 118:724-34. doi: 10.1016/S0016-5085(00)70142-9

26. Furusawa Y, Obata Y, Fukuda S, Endo TA, Nakato G, Takahashi D, et al. Commensal microbe-derived butyrate induces the differentiation of colonic regulatory T cells. Nature. (2013) 504:446-50. doi: 10.1038/nature12721

27. Stewart CJ, Ajami NJ, O'Brien JL, Hutchinson DS, Smith DP, Wong MC, et al. Temporal development of the gut microbiome in early childhood from the TEDDY study. Nature. (2018) 562:583-8. doi: 10.1038/s41586-018-0617-x

28. Bäckhed F, Roswall J, Peng Y, Feng Q, Jia H, Kovatcheva-Datchary P, et al. Dynamics and stabilization of the human gut microbiome during the first year of life. Cell Host Microbe. (2015) 17:690-703. doi: 10.1016/j.chom.2015.04.004

29. Nakayama J, Yamamoto A, Palermo-Conde LA, Higashi K, Sonomoto K, Tan J, et al. Impact of westernized diet on gut microbiota in children on Leyte Island. Front Microbiol. (2017) 8:197. doi: 10.3389/fmicb.2017.00197

30. Scheepers LEJM, Penders J, Mbakwa CA, Thijs C, Mommers M, Arts ICW. The intestinal microbiota composition and weight development in children: the KOALA Birth Cohort Study. Int J Obesity. (2015) 39:16-25 doi: $10.1038 /$ ijo. 2014.178

31. Lin A, Bik EM, Costello EK, Dethlefsen L, Haque R, Relman DA, et al. Distinct distal gut microbiome diversity and composition in healthy children from Bangladesh and the United States. PLoS ONE. (2013) 8:e53838. doi: 10.1371/journal.pone.0053838

32. Kolodziejczyk AA, Zheng D, Elinav E. Diet-microbiota interactions and personalized nutrition. Nat Rev Microbiol. (2019) 17:742-53. doi: 10.1038/s41579-019-0256-8

33. Aleksandrova K, Romero-Mosquera B, Hernandez V. Diet, gut microbiome and epigenetics: emerging links with inflammatory bowel diseases and prospects for management and prevention. Nutrients. (2017) 9:962. doi: 10.3390/nu9090962

34. Aas JA, Paster BJ, Stokes LN, Olsen I, Dewhirst FE. Defining the normal bacterial flora of the oral cavity. J Clin Microbiol. (2005) 43:5721-32. doi: 10.1128/JCM.43.11.5721-5732.2005

35. Ghannoum MA, Jurevic RJ, Mukherjee PK, Cui F, Sikaroodi M, Naqvi A, et al. Characterization of the oral fungal microbiome (mycobiome) in healthy individuals. PLoS Pathog. (2010) 6:e1000713. doi: 10.1371/journal.ppat.1000713

36. Dupuy AK, David MS, Li L, Heider TN, Peterson JD, Montano EA, et al. Redefining the human oral mycobiome with improved practices in ampliconbased taxonomy: discovery of malassezia as a prominent commensal. PLoS ONE. (2014) 9:e90899. doi: 10.1371/journal.pone.0090899

37. Dzidic M, Collado MC, Abrahamsson T, Artacho A, Stensson M, Jenmalm $\mathrm{MC}$, et al. Oral microbiome development during childhood: an ecological succession influenced by postnatal factors and associated with tooth decay. ISME J. (2018) 12:2292-306. doi: 10.1038/s41396-018-0204-z

38. Crielaard W, Zaura E, Schuller AA, Huse SM, Montijn RC, Keijser BJ. Exploring the oral microbiota of children at various developmental stages of their dentition in the relation to their oral health. BMC Med Genom. (2011) 4:22. doi: 10.1186/1755-8794-4-22

39. Mashima I, Theodorea CF, Thaweboon B, Thaweboon S, Scannapieco FA, Nakazawa F. Exploring the salivary microbiome of children stratified by the oral hygiene index. PLOS ONE. (2017) 12:1. 15. doi: 10.1371/journal.pone.0185274

40. Jesus VC de, Shikder R, Oryniak D, Mann K, Alamri A, Mittermuller B, et al. Sex-based diverse plaque microbiota in children with severe caries. J Dent Res. (2020) 99:703-12. doi: 10.1177/0022034520908595

41. Nearing JT, DeClercq V, Limbergen JV, Langille MGI. Assessing the variation within the oral microbiome of healthy adults. mSphere. (2020) 5:e00451-20. doi: $10.1128 / \mathrm{mSphere.00451-20}$

42. Woo VL. Oral manifestations of crohn's disease: a case report and review of the literature. Case Reports Dent. (2015) 2015:830472. doi: $10.1155 / 2015 / 830472$

43. Harikishan G, Reddy NR, Prasad H, Anitha S. Oral Crohn's disease without intestinal manifestations. J Pharm Bioallied Sci. (2012) 4:S431-4. doi: 10.4103/0975-7406.100322

44. Padmavathi B, Sharma S, Astekar M, Rajan Y, Sowmya G. Oral crohn's disease. J Oral Maxillofac Pathol. (2014) 18:S139-42. doi: 10.4103/0973-029X.141369

45. Feuerstein JD, Cheifetz AS. Crohn disease: epidemiology, diagnosis, and management. Mayo Clin Proc. (2017) 92:1088-103. doi: 10.1016/j.mayocp.2017.04.010

46. Cheifetz AS. Management of active Crohn disease. JAMA. (2013) 309:2150-8. doi: 10.1001/jama.2013.4466

47. Hussey S, Fleming P, Rowland M, Harty S, Chan L, Broderick A, et al. Disease outcome for children who present with oral manifestations of Crohn's disease. Eur Arch Paediatr Dent. (2012) 12:167-9. doi: 10.1007/BF03262800

48. Rowland M, Fleming P, Bourke B. Looking in the mouth for crohn's disease. Inflamm Bowel Dis. (2010) 16:332-7. doi: 10.1002/ibd.20983

49. Lankarani KB, Sivandzadeh GR, Hassanpour S. Oral manifestation in inflammatory bowel disease: a review. World J Gastroentero. (2013) 19:8571-9. doi: 10.3748/wjg.v19.i46.8571

50. Lourenço SV, Hussein TP, Bologna SB, Sipahi AM, Nico MMS. Oral manifestations of inflammatory bowel disease: a review based on the observation of six cases. J Eur Acad Dermatol Venereol. (2009) 24:204-7. doi: 10.1111/j.1468-3083.2009.03304.x

51. Docktor MJ, Paster BJ, Abramowicz S, Ingram J, Wang YE, Correll M, et al. Alterations in diversity of the oral microbiome in pediatric inflammatory bowel disease. Inflamm Bowel Dis. (2012) 18:935-42. doi: 10.1002/ibd.21874 
52. Crippa R, Zuccotti GV, Mantegazza C. Oral manifestations of gastrointestinal diseases in children. Part 2: Crohn's disease. Eur J Paediatr Dent Official J Eur Acad Paediatr Dent. (2016) 17:164-6. Available online at: http://admin.ejpd. eu/download/EJPD_2016_2ClinicalF.pdf

53. Katsanos KH, Torres J, Roda G, Brygo A, Delaporte E, Colombel J-F. Review article: non-malignant oral manifestations in inflammatory bowel diseases. Aliment Pharm Therap. (2015) 42:40-60. doi: 10.1111/apt.13217

54. Campbell H, Escudier M, Patel P, Nunes C, Elliott TR, Barnard K, et al. Distinguishing orofacial granulomatosis from Crohn's disease; two separate disease entities. Inflamm Bowel Dis. (2011) 17:2109-15. doi: 10.1002/ibd.21599

55. James J, Patton DW, Lewis CJ, Kirkwood EM, Ferguson MM. Oro-facial granulomatosis and clinical atopy. J Oral Med. (1986) 41:29-30.

56. Patel P, Barone F, Nunes C, Boursier L, Odell E, Escudier M, et al. Subepithelial dendritic B cells in orofacial granulomatosis. Inflamm Bowel Dis. (2010) 16:1051-60. doi: 10.1002/ibd.21169

57. Manichanh C, Rigottier-Gois L, Bonnaud E, Gloux K, Pelletier E, Frangeul L, et al. Reduced diversity of faecal microbiota in crohn's disease revealed by a metagenomic approach. Gut. (2006) 55:205-11. doi: 10.1136/gut.2005.073817

58. Joossens M, Huys G, Cnockaert M, Preter VD, Verbeke K, Rutgeerts P, et al. Dysbiosis of the faecal microbiota in patients with Crohn's disease and their unaffected relatives. Gut. (2011) 60:631-7. doi: 10.1136/gut.2010.223263

59. Gevers D, Kugathasan S, Denson LA, Vázquez-Baeza Y, Treuren WV, Ren B, et al. The treatment-naive microbiome in new-onset crohn's disease. Cell Host Microbe. (2014) 15:382-92. doi: 10.1016/j.chom.2014.02.005

60. Mottawea W, Chiang C-K, Mühlbauer M, Starr AE, Butcher J, Abujamel $\mathrm{T}$, et al. Altered intestinal microbiota-host mitochondria crosstalk in new onset Crohn's disease. Nat Commun. (2016) 7:ncomms13419. doi: 10.1038/ncomms13419

61. Said HS, Suda W, Nakagome S, Chinen H, Oshima K, Kim S, et al. Dysbiosis of salivary microbiota in inflammatory bowel disease and its association with oral immunological biomarkers. DNA Res. (2014) 21:15-25. doi: 10.1093/dnares/dst037

62. Schirmer M, Denson L, Vlamakis H, Franzosa EA, Thomas S, Gotman NM, et al. Compositional and temporal changes in the gut microbiome of pediatric ulcerative colitis patients are linked to disease course. Cell Host Microbe. (2018) 24:600-10.e4. doi: 10.1016/j.chom.2018.09.009

63. Seksik P, Rigottier-Gois L, Gramet G, Sutren M, Pochart P, Marteau P, et al. Alterations of the dominant faecal bacterial groups in patients with crohn's disease of the colon. Gut. (2003) 52:237-42. doi: 10.1136/gut.52.2.237

64. Morgan XC, Tickle TL, Sokol H, Gevers D, Devaney KL, Ward DV, et al. Dysfunction of the intestinal microbiome in inflammatory bowel disease and treatment. Genome Biol. (2012) 13:R79. doi: 10.1186/gb-2012-13-9-r79

65. Shaw KA, Bertha M, Hofmekler T, Chopra P, Vatanen T, Srivatsa A, et al. Dysbiosis, inflammation, and response to treatment: a longitudinal study of pediatric subjects with newly diagnosed inflammatory bowel disease. Genome Med. (2016) 8:75. doi: 10.1186/s13073-016-0331-y

66. Olbjørn C, Småstuen MC, Thiis-Evensen E, Nakstad B, Vatn MH, Jahnsen J, et al. Fecal microbiota profiles in treatment-naïve pediatric inflammatory bowel disease - associations with disease phenotype, treatment, and outcome. Clin Exp Gastroenterol. (2019) 12:37-49. doi: 10.2147/CEG.S186235

67. Wang Y, Gao X, Ghozlane A, Hu H, Li X, Xiao Y, et al. Characteristics of fecal microbiota in pediatric crohn's disease and their dynamic changes during infliximab therapy. J Crohns Colitis. (2017) 12:337-46. doi: 10.1093/ecco-jcc/jjx153

68. Halfvarson J, Brislawn CJ, Lamendella R, Vázquez-Baeza Y, Walters WA, Bramer LM, et al. Dynamics of the human gut microbiome in inflammatory bowel disease. Nat Microbiol. (2017) 2:17004. doi: 10.1038/nmicrobiol.2017.4

69. Knights D, Silverberg MS, Weersma RK, Gevers D, Dijkstra G, Huang H, et al. Complex host genetics influence the microbiome in inflammatory bowel disease. Genome Med. (2014) 6:107. doi: 10.1186/s13073-014-0107-1

70. Li E, Zhang Y, Tian X, Wang X, Gathungu G, Wolber A, et al. Influence of crohn's disease related polymorphisms in innate immune function on ileal microbiome. PLoS ONE. (2019) 14:e0213108. doi: 10.1371/journal.pone.0213108

71. Li Q, Wang C, Tang C, He Q, Li N, Li J. Dysbiosis of gut fungal microbiota is associated with mucosal inflammation in crohn's disease.
J Clin Gastroenterol. (2014) 48:513-23. doi: 10.1097/MCG.0000000000 000035

72. Chehoud C, Albenberg LG, Judge C, Hoffmann C, Grunberg S, Bittinger $\mathrm{K}$, Bet al. Fungal signature in the gut microbiota of pediatric patients with inflammatory bowel disease. Inflamm Bowel Dis. (2015) 21:1948-56. doi: 10.1097/MIB.0000000000000454

73. Kumamoto CA. The fungal mycobiota: small numbers, large impacts. Cell Host Microbe. (2016) 19:750-1. doi: 10.1016/j.chom.2016.05.018

74. Mukhopadhya I, Hansen R, Meharg C, Thomson JM, Russell RK, Berry $\mathrm{SH}$, et al. The fungal microbiota of de-novo paediatric inflammatory bowel disease. Microbes Infect Institut Pasteur. (2014) 17:304-10. doi: 10.1016/j.micinf.2014.12.001

75. Sokol H, Leducq V, Aschard H, Pham H-P, Jegou S, Landman C, et al. Fungal microbiota dysbiosis in IBD. Gut. (2016) 66:gutjnl-2015-310746. doi: 10.1136/gutjnl-2015-310746

76. Limon JJ, Tang J, Li D, Wolf AJ, Michelsen KS, Funari V, et al. Malassezia is associated with crohn's disease and exacerbates colitis in mouse models. Cell Host Microbe. (2019) 25:377-88.e6. doi: 10.1016/j.chom.2019.01.007

77. He J, Li Y, Cao Y, Xue J, Zhou X. The oral microbiome diversity and its relation to human diseases. Folia Microbiol. (2014) 60:69-80. doi: 10.1007/s12223-014-0342-2

78. Xun Z, Zhang Q, Xu T, Chen N, Chen F. Dysbiosis and ecotypes of the salivary microbiome associated with inflammatory bowel diseases and the assistance in diagnosis of diseases using oral bacterial profiles. Front Microbiol. (2018) 9:1136. doi: 10.3389/fmicb.2018.01136

79. Zhang T, Kayani M ur R, Hong L, Zhang C, Zhong J, Wang Z, et al. Dynamics of the salivary microbiome during different phases of crohn's disease. Front Cell Infect Microbiol. (2020) 10:544704. doi: 10.3389/fcimb.2020. 544704

80. Goel RM, Prosdocimi EM, Amar A, Omar Y, Escudier MP, Sanderson JD, et al. Streptococcus salivarius: a potential salivary biomarker for orofacial granulomatosis and crohn's disease? Inflamm Bowel Dis. (2019) 25:izz022. doi: $10.1101 / 422865$

81. Derrien M, Vlieg JET van H. Fate, activity, and impact of ingested bacteria within the human gut microbiota. Trends Microbiol. (2015) 23:354-66. doi: 10.1016/j.tim.2015.03.002

82. Auchtung TA, Fofanova TY, Stewart CJ, Nash AK, Wong MC, Gesell JR, et al. Investigating colonization of the healthy adult gastrointestinal tract by fungi. mSphere. (2018) 3:e00092-18. doi: 10.1128/mSphere.00092-18

83. Larsen JM, Steen-Jensen DB, Laursen JM, Søndergaard JN, Musavian HS, Butt $\mathrm{TM}$, et al. Divergent pro-inflammatory profile of human dendritic cells in response to commensal and pathogenic bacteria associated with the airway microbiota. PLoS ONE. (2012) 7:e31976. doi: 10.1371/journal.pone.0031976

84. Amer A, Whelan A, Al-Hebshi NN, Healy CM, Moran GP. Acetaldehyde production by Rothia mucilaginosa isolates from patients with oral leukoplakia. J Oral Microbiol. (2020) 12:1743066. doi: 10.1080/20002297.2020.1743066

85. Rengarajan S, Vivio EE, Parkes M, Peterson DA, Roberson EDO, Newberry RD, et al. Dynamic immunoglobulin responses to gut bacteria during inflammatory bowel disease. Gut Microbes. (2019) 11:1-16. doi: 10.1080/19490976.2019.1626683

86. Atarashi K, Suda W, Luo C, Kawaguchi T, Motoo I, Narushima S, et al. Ectopic colonization of oral bacteria in the intestine drives TH1 cell induction and inflammation. Science. (2017) 358:359-65. doi: 10.1126/science. aan 4526

Conflict of Interest: The authors declare that the research was conducted in the absence of any commercial or financial relationships that could be construed as a potential conflict of interest.

Copyright (C) 2021 Elmaghrawy, Hussey and Moran. This is an open-access article distributed under the terms of the Creative Commons Attribution License (CC BY). The use, distribution or reproduction in other forums is permitted, provided the original author(s) and the copyright owner(s) are credited and that the original publication in this journal is cited, in accordance with accepted academic practice. No use, distribution or reproduction is permitted which does not comply with these terms. 